\title{
Analytical soft-core potentials for macromolecular fluids and mixtures
}

\author{
G. Yatsenko, E. J. Sambriski, M. A. Nemirovskaya, M. Guenza \\ Institute of Theoretical Science, Department of Chemistry, University of Oregon, Eugene, OR 97403
}

\begin{abstract}
An analytical description of polymer melts and their mixtures as liquids of interacting soft colloidal particles is obtained from liquid-state theory. The derived center-of-mass pair correlation functions with no adjustable parameters reproduce those computed from united atom molecular dynamics simulations. The coarse-grained description correctly bridges micro- and mesoscopic fluid properties. Molecular dynamics simulations of soft colloidal particles interacting through the calculated effective pair potentials are consistent with data from microscale simulations and analytical formulas.
\end{abstract}

The formulation of an accurate mesoscopic description of macromolecular fluids has been a longstanding goal in polymer physics. Experimentally-relevant polymer dynamics span a wide range of timescales, for which large-scale, long-time properties still depend strongly on the local molecular structure [1]. Pertinent information on structure and dynamics of polymer liquids has been gained from united atom (UA) molecular dynamics (MD) simulations. However, the MD computational time increases as the squared number of interacting units, and the latter has to be large to approximate the thermodynamic limit, rendering an all-atom simulation of long-time polymer dynamics a prohibitive task. One strategy devised to overcome this problem is to renormalize the liquid structure and dynamics using effective-unit coarse-grained descriptions 1]. Specifically, polymers can be described mesoscopically as soft interpenetrating spheres having the overall size of the polymer, i.e., the radius of gyration $R_{g}$. However, to correctly perform the renormalization procedure, a theoretical framework that bridges properly different lengthscales of interest is needed. Phenomenological mesoscopic potentials were implemented by Dautenhahn and Hall, and later on by Murat and Kremers, to describe polymer melts and blends [2, 3]. Hansen and coworkers have recently developed a rigorous numerical description of polymer solutions as liquids of soft interacting colloidal particles 4].

In this Letter we start from first-principles liquid-state theory and derive an analytical form of center-of-mass (c.o.m.) pair correlation functions, from which the effective pair soft-core potential acting between molecules in polymer liquids (melts) and their mixtures (blends) is obtained. The c.o.m. pair correlation functions reproduce mesoscale liquid structures obtained from UA-MD simulations [5, 6, 7] without adjustable parameters. Test systems are polymer melts with different architecture, local semiflexibility, and degree of polymerization (Table I), as well as their mixtures (Table II). Finally, the mesoscopic potential derived by an inversion procedure is used in MD simulations of soft colloidal particles, which reproduce the liquid structure at the level of c.o.m. pair correlation functions.

The renormalized pair interaction potential is a function of the c.o.m. total pair correlation function $h(r)$. In reciprocal space, $h(k)=\left[\omega^{m C}(k) / \omega^{m m}(k)\right]^{2} h^{m m}(k)$, after a procedure devised by Krakoviack, Hansen, and Louis [8]. Here, $\omega^{m C}(k)$ is the intrachain monomer distribution about the c.o.m. Also, $h^{m m}(k)$ and $\omega^{m m}(k)$ are the monomermonomer intermolecular total pair and intramolecular correlation functions, respectively. We assume a Gaussian description for intramolecular form factors: $\omega^{m m}(k)=N /\left(1+k^{2} R_{g}^{2} / 2\right)$, where $N$ is the number of "monomeric" units of length $\sigma$ and $R_{g}=\sqrt{N / 6} \sigma$ is the overall chain size, and $\omega^{m C}(k)=N e^{-k^{2} R_{g}^{2} / 6}\left[\underline{9}\right.$. For $h^{m m}(k)$, we use the thread-limit PRISM description [10], in which the chain is treated as an infinite thread of vanishing thickness. Since this model correctly reproduces the appearance of correlation hole effects on the scale of $R_{g}$, a distinguishing feature of macromolecular fluids, liquid properties at the mesoscale of interest should be described well. Now, $h^{m m}(k)=$ $4 \pi \xi_{\rho}^{\prime}\left[\xi_{\rho}^{2} /\left(1+k^{2} \xi_{\rho}^{2}\right)-\xi_{c}^{2} /\left(1+k^{2} \xi_{c}^{2}\right)\right]$, where $\xi_{c}=R_{g} / \sqrt{2}$ is the correlation hole lengthscale. The density fluctuation length is defined as $\xi_{\rho}^{-1}=\xi_{c}^{-1}+\xi_{\rho}^{\prime-1}$ with $\xi_{\rho}^{\prime}=R_{g} /\left(2 \pi \rho_{c h}^{*}\right)$, and $\rho_{c h}^{*}=\rho_{c h} R_{g}^{3}$, where $\rho_{c h}$ is the chain number density. In real space, we find

$$
\begin{aligned}
h(r)= & \frac{3}{4} \sqrt{\frac{3}{\pi}} \frac{\xi_{\rho}^{\prime}}{R_{g}}\left(1-\frac{\xi_{c}^{2}}{\xi_{\rho}^{2}}\right) e^{-3 r^{2} /\left(4 R_{g}^{2}\right)}-\frac{1}{2} \frac{\xi_{\rho}^{\prime}}{r}\left(1-\frac{\xi_{c}^{2}}{\xi_{\rho}^{2}}\right)^{2} e^{R_{g}^{2} /\left(3 \xi_{\rho}^{2}\right)} \\
& \times\left[e^{r / \xi_{\rho}} \operatorname{erfc}\left(\frac{R_{g}}{\xi_{\rho} \sqrt{3}}+\frac{r \sqrt{3}}{2 R_{g}}\right)-e^{-r / \xi_{\rho}} \operatorname{erfc}\left(\frac{R_{g}}{\xi_{\rho} \sqrt{3}}-\frac{r \sqrt{3}}{2 R_{g}}\right)\right],
\end{aligned}
$$

where $\operatorname{erfc}(x)$ is the complementary error function. This expression satisfies the condition $h(r) \geq-1$, spanning melt to dilute solution densities up to $\rho_{c h}^{*} \geq 0.03$. Eq. (11) correctly recovers the isothermal compressibility $\kappa_{T}$ [11] related to the $k \rightarrow 0$ limit of the mesoscale static structure factor as $S(0)=1+\rho_{c h} h(0)=\left(\xi_{\rho} / \xi_{c}\right)^{2}=\rho_{c h} k_{B} T \kappa_{T}$.

Our total correlation function $h(r)$ effectively maps the polymeric liquid onto a fluid of soft interpenetrating colloids of radius $R_{g}$ and density $\rho_{c h}$. Translated into the colloidal-particle framework, Eq. (10) is a function of the reduced variables $\tilde{r} \equiv r / R_{g}, \tilde{\xi}_{\rho}^{\prime}=\xi_{\rho}^{\prime} / R_{g} \equiv\left(2 \pi \rho_{c h}^{*}\right)^{-1}$, and $\tilde{\xi}_{\rho}=\xi_{\rho} / R_{g} \equiv\left[\sqrt{2}\left(1+\pi \sqrt{2} \rho_{c h}^{*}\right)\right]^{-1}$. Then, Eq. (11) reduces to 
$h(r) \equiv h\left(\tilde{r}, \tilde{\xi}_{\rho}\right)$, where we define

$$
\begin{aligned}
h\left(\tilde{r}, \tilde{\xi}_{\lambda}\right)= & \frac{3}{4} \sqrt{\frac{3}{\pi}} \tilde{\xi}_{\rho}^{\prime}\left(1-\frac{1}{2 \tilde{\xi}_{\lambda}^{2}}\right) e^{-3 \tilde{r}^{2} / 4}-\frac{1}{2} \frac{\tilde{\xi}_{\rho}^{\prime}}{\tilde{r}}\left(1-\frac{1}{2 \tilde{\xi}_{\lambda}^{2}}\right)^{2} e^{1 /\left(3 \tilde{\xi}_{\lambda}^{2}\right)} \\
& \times\left[e^{\tilde{r} / \tilde{\xi}_{\lambda}} \operatorname{erfc}\left(\frac{1}{\tilde{\xi}_{\lambda} \sqrt{3}}+\frac{\tilde{r} \sqrt{3}}{2}\right)-e^{-\tilde{r} / \tilde{\xi}_{\lambda}} \operatorname{erfc}\left(\frac{1}{\tilde{\xi}_{\lambda} \sqrt{3}}-\frac{\tilde{r} \sqrt{3}}{2}\right)\right] .
\end{aligned}
$$

We tested Eq. (11) against UA-MD simulations for polymer melts of polyethylene (PE) for increasing degree of polymerization $(N=44,66,96)[5]$ as well as different local structure and flexibility, including syndiotactic (sPP), isotactic (iPP) and head-to-head (hhPP) polypropylenes [6, 7]. Input parameters to the theory were site number density $\rho$, temperature $T$, and $R_{g}$ from Table I. Our analytical expression agrees well with $h(r)$ from simulations within statistical error (Figs. 1 and 2). Specifically, for PE melts it reproduces the tendency for chains to interpenetrate more efficiently with increasing length, an effect due to the fractal dimension of polymer chains. For slightly branched polymer melts, the number of interpolymer contacts becomes larger with increasing polymer stiffness (increasing $R_{g}$ at constant $N$ ). Good agreement between theory and computer simulations is found also in reciprocal space, where the analytical Fourier transform of Eq. (11) reproduces simulation data in the entire range for $k \geq R_{g}^{-1}$ (Fig. 3).

A simplified form of Eq. (11) can be derived in the limit of long polymer chains $\left(\tilde{\xi}_{\rho} \rightarrow 0\right)$ as is implicitly assumed by the use of Gaussian form factors, for which we find

$$
h\left(\tilde{r}, \tilde{\xi}_{\rho}\right) \approx-\frac{39}{16} \sqrt{\frac{3}{\pi}} \tilde{\xi}_{\rho}\left(1+\sqrt{2} \tilde{\xi}_{\rho}\right)\left(1-\frac{9 \tilde{r}^{2}}{26}\right) e^{-3 \tilde{r}^{2} / 4},
$$

where $h\left(\tilde{r}, \tilde{\xi}_{\rho}\right) / \tilde{\xi}_{\rho}$ is a universal function of the reduced distance $\tilde{r}$ up to the second-order correction in $\tilde{\xi}_{\rho}$. Eq. (3) is a good approximation for Eq. (1): results obtained with the two expressions cannot be distinguished in Fig. 1. Both equations recover the correct trend of greater chain interpenetration with increasing $R_{g}$ and/or liquid density as $h(0) \propto\left(\rho_{c h}^{*}\right)^{-1}$.

From Eq. (11), the effective potential is calculated self-consistently using an inversion procedure based on the HNC approximation, $\beta v(r)=h(r)-\ln [h(r)+1]-c(r)$, where $\beta=1 / k_{B} T$. The direct correlation function $c(r)$ [1] is obtained from the Fourier transform of the Ornstein-Zernike equation $c(k)=h(k) /\left[1+\rho_{c h} h(k)\right]$. While the HNC closure is known to work well for dilute colloidal systems, its applicability is questionable in dense systems where many-body interactions should be important 11]. However, the pair HNC interaction potential works well for the mesoscopically-renormalized polymer melts investigated here. We performed constant temperature MD simulations of a liquid of soft colloidal particles of radius $R_{g}$ interacting through the derived pair potential, for chains of $N=44$, 66 , and 96 units. Corresponding effective potentials $\beta v(r)$ as functions of the normalized distance $r / R_{g}$ differ slightly for the three cases. The potential for $N=44$ is shown in the inset of Fig. 1. Mesoscale simulations recover the liquid structure obtained in UA-MD simulations in both real (Fig. 1) and reciprocal space (Fig. 3).

We extended the formalism just presented to treat binary polymer mixtures. The homopolymer species are $A$ and $B$, characterized by radii of gyration $R_{g A}$ and $R_{g B}$, with number of chain units $N_{A}$ and $N_{B}$, and unit lengths $\sigma_{A}=\sqrt{6 / N_{A}} R_{g A}$ and $\sigma_{B}=\sqrt{6 / N_{B}} R_{g B}$. The volume fraction of component $A$ is given by $\phi$ and $\gamma=\sigma_{B} / \sigma_{A}$ represents the mismatch in local chain semiflexibility. For a generic pair $\{\alpha, \beta \in(A, B)\}$, we have $h_{\alpha \beta}(k)=\left[\omega_{\alpha}^{m C}(k) \omega_{\beta}^{m C}(k) /\left(\omega_{\alpha}^{m m}(k) \omega_{\beta}^{m m}(k)\right)\right]^{2} h_{\alpha \beta}^{m m}(k)$. Chains are assumed to obey Gaussian intramolecular distributions with $\omega^{m C}(k)$ and $\omega^{m m}(k)$ defined as in the melt case. The $h_{\alpha \beta}^{m m}(k)$ follow the PRISM-blend thread model described by Tang and Schweizer [12], and is extended here to include asymmetries in local chemical structure and flexibility $\left(\sigma_{A} \leq \sigma_{B}\right)$. The advantage of this approach is that no closure approximations need to be invoked, while the miscibility parameter $\chi$ enters as an input to the theory [13]. This allows us to describe the renormalized structure and potential for systems following either upper or lower critical solution temperature phase diagrams within one theoretical framework.

On applying inverse Fourier transforms, the blend $h_{\alpha \beta}(k)$ in real space are given by

$$
\begin{aligned}
& h_{A A}(r)=\frac{1-\phi}{\phi} I_{A A}^{\phi}(r)+\gamma^{2} I_{A A}^{\rho}(r), \quad h_{B B}(r)=\frac{\phi}{1-\phi} I_{B B}^{\phi}(r)+\frac{1}{\gamma^{2}} I_{B B}^{\rho}(r), \\
& h_{A B}(r)=-I_{A B}^{\phi}(r)+I_{A B}^{\rho}(r),
\end{aligned}
$$

with

$$
I_{\alpha \beta}^{\lambda}(r)=\frac{3}{4} \sqrt{\frac{3}{\pi}} \frac{\xi_{\rho}^{\prime}}{R_{g \alpha \beta}} \vartheta_{\alpha \beta 1}\left(1-\frac{\xi_{c \alpha \beta}^{2}}{\xi_{\lambda}^{2}}\right) e^{-3 r^{2} /\left(4 R_{g \alpha \beta}^{2}\right)}-\frac{1}{2} \frac{\xi_{\rho}^{\prime}}{r} \vartheta_{\alpha \beta 2}\left(1-\frac{\xi_{c \alpha \beta}^{2}}{\xi_{\lambda}^{2}}\right)^{2} e^{R_{g \alpha \beta}^{2} /\left(3 \xi_{\lambda}^{2}\right)}
$$




$$
\times\left[e^{r / \xi_{\lambda}} \operatorname{erfc}\left(\frac{R_{g \alpha \beta}}{\xi_{\lambda} \sqrt{3}}+\frac{r \sqrt{3}}{2 R_{g \alpha \beta}}\right)-e^{-r / \xi_{\lambda}} \operatorname{erfc}\left(\frac{R_{g \alpha \beta}}{\xi_{\lambda} \sqrt{3}}-\frac{r \sqrt{3}}{2 R_{g \alpha \beta}}\right)\right] .
$$

Here $\xi_{\lambda}$ for $\{\lambda \in(\rho, \phi)\}$ identifies the lengthscale for density or concentration fluctuation correlations. The concentration fluctuation length $\xi_{\phi}=\sigma_{A B} / \sqrt{24\left(\chi_{s}-\chi\right) \phi(1-\phi)}$ diverges at the spinodal temperature where $\chi=\chi_{s}$. The average segment length is $\sigma_{A B}^{2}=\phi \sigma_{B}^{2}+(1-\phi) \sigma_{A}^{2}$, while $R_{g \alpha \beta} \equiv \sqrt{\left(R_{g \alpha}^{2}+R_{g \beta}^{2}\right) / 2}=\xi_{c \alpha \beta} \sqrt{2}$, with $\xi_{c \alpha \beta}$ being the average correlation hole length. Also, $\vartheta_{\alpha \beta 1}=\left[1-\xi_{c \alpha \alpha}^{2} \xi_{c \beta \beta}^{2} /\left(\xi_{c \alpha \beta}^{2} \xi_{\lambda}^{2}\right)\right] /\left[1-\xi_{c \alpha \beta}^{2} / \xi_{\lambda}^{2}\right]$ and $\vartheta_{\alpha \beta 2}=\left[\left(1-\xi_{c \alpha \alpha}^{2} / \xi_{\lambda}^{2}\right)\left(1-\xi_{c \beta \beta}^{2} / \xi_{\lambda}^{2}\right)\right] /\left[1-\xi_{c \alpha \beta}^{2} / \xi_{\lambda}^{2}\right]^{2}$. The self terms in the density fluctuation contributions $I_{\alpha \alpha}^{\rho}(r)$ are formally identical to the total pair correlation function in the melt. For a totally symmetric blend $\left(N_{A}=N_{B}\right.$ with $\sigma_{A}=\sigma_{B}$ ), Eqs. (45) correctly recover the melt equation in the athermal limit where enthalpic effects are negligible.

We tested Eqs. (45) against UA-MD simulations of blends [6, 7] with polymer components having different degree of polymerization $N$, local semiflexibility/branching $\sigma$, and volume fraction $\phi$. By convention, the $B$-component is assumed to be the stiffest. In Fig. 4, we report only two representative examples (see Table II). For a thermal $\phi$-symmetric mixture of linear (PE) and slightly branched (hhPP) molecules, the theory agrees well with simulations $\left(\chi=0.0016\right.$ Ref. [13] and $\left.\chi_{s}=0.0211\right)$. The stiffest component $(\mathrm{PE})$ shows a higher number of intermolecular contacts than the flexible one (hhPP). A weak self-clustering of the most flexible species is observed at a distance comparable to the polymer size, while the number of $A B$ contacts is still large at this temperature, an indication that the system is far from its phase transition. Similar effects are shown for the second system in Fig. 4, which is a thermal $\phi$-asymmetric blend of slightly branched (iPP) and linear $(\mathrm{PE})$ molecules $\left(\chi=0.005\right.$ Ref. [6] and $\left.\chi_{s}=0.0281\right)$. In asymmetric mixtures, the minority species (iPP) presents an enhanced clustering, in agreement with experimental data and simulations $[6,7]$.

The blend pair correlation functions can be mapped onto a system of interacting soft colloids of dimension $R_{g A}$ and $R_{g B}$, with chain volume fraction given by $\phi=\phi_{c h}$ and mismatch in chain size given by $\gamma=R_{g B} / R_{g A}$. The analytical equations are considerably simplified for symmetric mixtures where $\gamma=1$, and we confine our presentation to this case [14]. For each pair interaction, Eq. (5) reduces to Eq. (2): $I^{\lambda}\left(\tilde{r}, \tilde{\xi}_{\lambda}\right) \equiv h\left(\tilde{r}, \tilde{\xi}_{\lambda}\right)$ where $\tilde{\xi}_{\lambda}$ for $\{\lambda \in(\rho, \phi)\}$ and $\tilde{\xi}_{\phi}=$ $\xi_{\phi} / R_{g} \equiv 1 / \sqrt{2\left(1-\chi / \chi_{s}\right)}$. By combining Eqs. (45) into density and concentration fluctuation contributions following Bhatia-Thornton's formalism [11], we recover known expressions for a mixture of symmetric colloidal particles (e.g., liquid alloys). For example, in the $k \rightarrow 0$ limit, the correlation in number density $S^{\rho \rho}(0)=\left(\xi_{\rho} / \xi_{c}\right)^{2}$ correctly recovers $\kappa_{T}$, while the concentration fluctuation correlation reduces to the known formula $S^{\phi \phi}(0)=\phi(1-\phi) /[1-2 \phi(1-\phi) N \chi]$ upon introducing Flory's definition of $\chi_{s}$. Finally, the cross term $S^{\rho \phi}(0)$ vanishes in agreement with the theory of symmetric particle mixtures [11]. Consistency between Eqs. (45) and known properties of colloidal mixtures supports our mapping of polymer blends onto a mixture of interacting soft colloidal particles.

Summarizing, we report here the derivation of an analytical renormalized description of polymer melts and blends as fluids of mesoscopic soft colloidal particles. The related soft-core Gaussian potential explicitly bridges meso- and microscale properties. The derived c.o.m. pair correlation functions reproduce, in both $r$ - and $k$-space, fluid structures obtained from UA-MD simulations. Using the melt potential calculated from the HNC approximation, we perform MD simulations of liquids comprised of soft colloidal particles, from which the obtained pair correlation functions correctly recover the liquid structure from UA-MD simulations, further supporting our renormalized analytical description of polymer liquids.

We are grateful to G. S. Grest and E. Jaramillo for sharing UA-MD simulation trajectories. We acknowledge support from the National Science Foundation under grant DMR-0207949. Also, EJS acknowledges support from an NSF Graduate Research Fellowship.

[1] D. Frenkel and B. Smit, Understanding Molecular Simulation (Academic, New York, 2000).

[2] J. Dautenhahn and C. Hall, Macromol. 27, 5399 (1994).

[3] M. Murat and K. Kremer, J. Chem. Phys. 108, 4340 (1998).

[4] P. G. Bolhuis, A. A. Louis, J.-P. Hansen, and E. J. Meijer, J. Chem. Phys. 114, 4296 (2001) and references therein.

[5] M. Mondello and G. S. Grest, J. Chem. Phys. 106, 9327 (1997).

[6] D. Heine, D. T. Wu, J. G. Curro, and G. S. Grest, J. Chem. Phys. 118, 914 (2003).

[7] E. Jaramillo, D. T. Wu, G. S. Grest, and J. G. Curro, J. Chem. Phys. 120, 8883 (2004).

[8] V. Krakoviack, J.-P. Hansen, and A. A. Louis, Europhys. Lett. 58, 53 (2002).

[9] H. Yamakawa, Modern Theory of Polymer Solutions (Harper and Row, New York, 1971).

[10] K. S. Schweizer and J. G. Curro, Adv. Chem. Phys. 98, 1 (1997).

[11] J.-P. Hansen and I. R. McDonald, Theory of Simple Liquids (Academic, London, 1991). 
[12] H. Tang and K. S. Schweizer, J. Chem. Phys. 105, 779 (1996).

[13] N. P. Balsara, in Physical Properties of Polymers Handbook, edited by J. E. Mark (AIP, New York, 1996$)$, p. 257.

[14] G. Yatsenko, E. J. Sambriski, M. A. Nemirovskaya, M. Guenza, J. Chem. Phys. (accepted). 
TABLE I. Polyolefin melts.

\begin{tabular}{|c|c|c|c|c|}
\hline \hline Polymer & $N$ & $T[\mathrm{~K}]$ & $\rho\left[\right.$ sites $\left./ \AA^{3}\right]$ & $R_{g}[\AA]$ \\
\hline $\mathrm{PE}$ & 44 & 400 & 0.0324 & 10.50 \\
$\mathrm{PE}$ & 66 & 448 & 0.0329 & 13.32 \\
$\mathrm{PE}$ & 96 & 453 & 0.0328 & 16.78 \\
$\mathrm{sPP}$ & 96 & 453 & 0.0328 & 13.93 \\
$\mathrm{hhPP}$ & 96 & 453 & 0.0336 & 13.54 \\
$\mathrm{iPP}$ & 96 & 453 & 0.0328 & 11.34 \\
\hline \hline
\end{tabular}


TABLE II. Polyolefin blends $\left(T=453 \mathrm{~K}\right.$ and $\left.N_{A}=N_{B}=96\right)$.

\begin{tabular}{|c|c|c|c|c|}
\hline \hline Blend $[\mathrm{A} / \mathrm{B}]$ & $\phi$ & $\rho\left[\right.$ sites $\left./ \AA^{3}\right]$ & $R_{g A}[\AA]$ & $\gamma$ \\
\hline $\mathrm{hhPP} / \mathrm{PE}$ & 0.50 & 0.0332 & 12.32 & 1.34 \\
$\mathrm{iPP} / \mathrm{PE}$ & 0.25 & 0.0328 & 11.35 & 1.47 \\
\hline \hline
\end{tabular}


FIG. 1. Plot of $h(r)$ against $r / R_{g}$ for PE melts. Theory (full lines) is compared with UA-MD (filled symbols) and mesoscale (open symbols) simulations for $N=96$ (squares), 66 (circles), and 44 (diamonds). Inset shows a plot of $\beta v(r)$ against $r / R_{g}$ for the $N=44$ case.

FIG. 2. Plot of $h(r)$ against $r / R_{g}$ for melts of different polyolefins. Theory (full lines) is compared with UA-MD simulations for $\mathrm{PE}$ (squares), sPP (circles), iPP (downward triangles). Inset shows hhPP (upward triangles).

FIG. 3. Plot of $h(k)$ against $k \geq R_{g}^{-1}$. Theory (full lines) is compared with UA-MD simulations (filled symbols) and mesoscale simulations (open symbols). Left panel shows PE melts with $N=$ 96 (squares), 66 (circles), and 44 (diamonds). Right panel displays melts of different polyolefins: PE (circles), sPP (squares), iPP (downward triangles); inset shows hhPP (upward triangles).

FIG. 4. Plot of $h(r)$ against $r$ for blends. Theoretical curves in athermal (solid lines) and thermal (dashed lines) limits are compared with $A A$ - (circles), $A B$ - (diamonds) and $B B$-terms (squares) of $h(r)$ from UA-MD simulations of representative $\phi$-symmetric (upper panel) and $\phi$ - asymmetric (lower panel) blends. 